\title{
Individualized quality of life benefit and cost-effectiveness estimates of proton therapy for patients with oropharyngeal cancer
}

\author{
N. Patrik Brodin ${ }^{1,2^{*}}$, Rafi Kabarriti ${ }^{1,2}$, Clyde B. Schechter ${ }^{4}$, Mark Pankuch ${ }^{3}$, Vinai Gondi ${ }^{3}$, Shalom Kalnicki ${ }^{2,6}$, \\ Madhur K. Garg ${ }^{1,2,5,6}$ and Wolfgang A. Tomé $1,2,7^{*}$
}

\begin{abstract}
Background: Proton therapy is a promising advancement in radiation oncology especially in terms of reducing normal tissue toxicity, although it is currently expensive and of limited availability. Here we estimated the individual quality of life benefit and cost-effectiveness of proton therapy in patients with oropharyngeal cancer treated with definitive radiation therapy (RT), as a decision-making tool for treatment individualization.
\end{abstract}

Methods and materials: Normal tissue complication probability models were used to estimate the risk of dysphagia, esophagitis, hypothyroidism, xerostomia and oral mucositis for 33 patients, comparing delivered photon intensitymodulated RT (IMRT) plans to intensity-modulated proton therapy (IMPT) plans. Quality-adjusted life years (QALYs) lost were calculated for each complication while accounting for patient-specific conditional survival probability and assigning quality-adjustment factors based on complication severity. Cost-effectiveness was modeled based on upfront costs of IMPT and IMRT, and the cost of acute and/or long-term management of treatment complications. Uncertainties in all model parameters and sensitivity analyses were included through Monte Carlo sampling.

Results: The incremental cost-effectiveness ratios (ICERs) showed considerable variability in the cost of QALYs spared between patients, with median $\$ 361,405 / \mathrm{Q} A L Y$ for all patients, varying from $\$ 54,477 / \mathrm{QALY}$ to $\$ 1,508,845 / \mathrm{Q} A \mathrm{LY}$ between individual patients. Proton therapy was more likely to be cost-effective for patients with p16-positive tumors ( $\$ 234,201 /$ QALY), compared to p16-negative tumors ( $\$ 516,297 /$ QALY). For patients with p16-positive tumors treated with comprehensive nodal irradiation, proton therapy is estimated to be cost-effective in $\geq 50 \%$ of sampled cases for $8 / 9$ patients at $\$ 500,000 / \mathrm{QALY}$, compared to $6 / 24$ patients who either have p16-negative tumors or receive unilateral neck irradiation.

Conclusions: Proton therapy cost-effectiveness varies greatly among oropharyngeal cancer patients, and highlights the importance of individualized decision-making. Although the upfront cost, societal willingness to pay and healthcare administration can vary greatly among different countries, identifying patients for whom proton therapy will have the greatest benefit can optimize resource allocation and inform prospective clinical trial design.

Keywords: Proton therapy, Quality of life, Cost-effectiveness, Individualized risk-assessment

*Correspondence: patrik.brodin@einsteinmed.org; wolfgang. tome@einsteinmed.org

1 Institute for Onco-Physics, Albert Einstein College of Medicine, Bronx, NY 10461, USA

Full list of author information is available at the end of the article

\section{Background}

Head and neck cancer (HNC) presents a major challenge in modern day radiation oncology, where radiation therapy (RT) is a critical component of an aggressive multidisciplinary treatment approach. Although effective as definitive therapy with 3-year loco-regional control rates 
in the range of $70-80 \%$ [1-6], HNC RT carries considerable risk for a large number of severe normal tissue complications. This substantial toxicity profile is usually ascribed to these cancers requiring very high doses of radiation and being anatomically situated in an area with many radiation-sensitive organs-at-risk (OARs). Thus, to deliver a sufficient radiation dose to the gross disease and involved lymph nodes the neighboring tissues will be subject to high doses of radiation. HNC constitutes a situation in which technological advances in RT have played a vital role in reducing the side effect profile, with the implementation of intensity-modulated RT (IMRT) being a key breakthrough.

The availability of proton radiation therapy constitutes another advance in the delivery of RT, and offers improved normal tissue sparing for a variety of cancer sites. The potential interest in HNC RT using protons is focused largely around reducing the risk of normal tissue toxicity. Proton therapy differs substantially from photon radiation therapy, characterized by much sharper distal dose fall-off, slightly wider penumbra, higher skin dose and different strategies to account for uncertainties in treatment delivery. Utilizing the proton therapy Bragg peak and a multi-beam approach, the goal is to reduce the dose to critical normal tissues, thereby reducing the risk of severe toxicity and improving the quality of life for HNC patients receiving definitive RT. Some of these strategies have been explored in previous modeling studies [7-11]. While results have been mixed as to the absolute benefit of proton therapy for $\mathrm{HNC}$, an important remaining question is whether this benefit is also costeffective, as proton therapy is currently expensive and of limited availability compared to standard photon RT.

We previously showed that the estimated quality of life benefit with proton therapy is highly variable between individual oropharyngeal HNC patients, suggesting that not all patients would receive a better treatment with protons [12]. Here, we performed a comprehensive cost-effectiveness analysis aiming to identify for which oropharyngeal cancer patients the benefit offered by proton therapy is estimated to be the most, or least, cost-effective.

\section{Methods and materials}

\section{Patient material and quality-adjusted life years (QALYs)} calculation

We previously published a quantitative decision-support strategy comparing the QALYs lost from normal tissue complications following definitive photon or proton RT for patients with oropharyngeal cancer [12]. Briefly, 33 oropharyngeal HNC patients treated with definitive photon IMRT at our institution were identified in accordance with an approved institutional review board protocol. Twenty-one patients were treated with bilateral comprehensive nodal irradiation and 12 patients with unilateral nodal irradiation. Comparative proton therapy treatment plans were generated based on clinical protocols from a collaborating institution using pencil beam scanning intensity-modulated proton therapy (IMPT) for HNC. Normal tissue complication probability (NTCP) models identified in a comprehensive systematic review [13] were used to calculate the estimated risk of dysphagia, esophagitis, xerostomia, hypothyroidism and oral mucositis for the photon and proton therapy plans, respectively.

Quality-adjustment factors (detailed in Additional file 1: Table S1) were then used to estimate the reduction in QALYs attributable to each complication, accounting for the age, sex and p16-status specific conditional survival probability. A detailed explanation of the proton therapy treatment planning along with NTCP and QALY calculations for these patients has been previously reported [12]. In short, the QALYs were calculated as the estimated survival time either free of any modeled normal tissue complications or spent in the corresponding health states of experiencing a given complication. Thus, the estimated QALYs depend on the probability of a given treatment complication, the estimated survival time, the duration of the given complication and the severity, which is represented by the quality-adjustment factors. A quality-adjustment factor (also referred to as utilities) of 1 would represent perfect health and 0 would represent death. This way, we can e.g. compare a mild complication that may last for a long time with a severe complication that only exists in an acute phase, using QALYs as a common scale metric.

\section{Cost-effectiveness analysis}

The cost-effectiveness of proton IMPT compared to photon IMRT for oropharyngeal HNC RT was estimated based on 33 treatment fractions. The upfront cost of photon and proton therapy was determined from the 2017 CMS average national Medicare physician fee schedule reimbursement rates. Quoted costs of $\$ 18,415$ for 30 fractions of complex IMRT and $\$ 27,772$ for 25 fractions of complex proton therapy were extrapolated to $\$ 20,257$ and $\$ 36,659$ as the upfront cost of 33 fractions of IMRT and IMPT, respectively.

The interventions and patient procedures related to the management of the normal tissue complications included in this analysis are based on the standard of care for HNC patients treated at our institution. Patients with acute grade $\geq 3$ oral mucositis receive long-acting pain medication in terms of a Fentanyl patch $25 \mu \mathrm{g} / \mathrm{h}$ and short-acting pain medication with 3-4 Percocet $325 \mathrm{mg}$ tablets per day. They also receive a daily mucositis cocktail consisting 
of Lidocaine $2 \%$ viscous solution, Maalox dissolvable tablet and Benadryl $50 \mathrm{mg} / \mathrm{ml}$ and once weekly IV hydration for 4 weeks. Patients with acute grade $\geq 3$ esophagitis receive similar management and a proportion of patients experiencing either of these side effects are also expected to require percutaneous endoscopic gastrostomy (PEG) tube placement $(\sim 30 \%)$, emergency room visits $(\sim 15 \%$ for oral mucositis and $\sim 10 \%$ for esophagitis), and $\sim 10 \%$ with these side effects require inpatient hospital admission. Patients with grade $\geq 3$ oral mucositis or esophagitis are also expected to miss about 1 month of work due to these debilitating side effects.

Patients experiencing grade $\geq 2$ dysphagia, which can be chronic in many cases, may require stricture dilation and this proportion was estimated to be about $15-17 \%$ of HNC patients according to previous studies $[14,15]$. A proportion of patients with grade $\geq 2$ dysphagia also require a chronic PEG tube $(\sim 10 \%)$. Patients experiencing chronic hypothyroidism (defined as elevated TSH $\mathrm{w} /$ or w/o T4/T3 changes requiring hormone replacement) will receive lifelong Levothyroxine at $1.6 \mu \mathrm{g} / \mathrm{kg}$ daily as recommended by Vaidya and Pearce [16]. We currently do not have an effective standard of care management for grade $\geq 2$ xerostomia and this is considered a chronic condition. For this analysis we used the suggested intervention from Siddiqui and Movsas of Pilocarpine $5 \mathrm{mg}$ three times per day for 8-12 weeks and Cevimeline 30-45 mg three times per day for 6 weeks [17].

The costs of any drug treatments were extracted from the US National Average Drug Acquisition Cost (NADAC) database (accessed March 2018) and costs for medical procedures, emergency room visits and inpatient hospitalization were extracted from US national statistics or published reports, summarized in Table 1 . The cost of lost income was based on the US median income per capita from 2018 census bureau statistics. Discounting was applied for the estimated QALYs, as well as cost of Levothyroxine therapy and chronic PEG tube dependency, as these require long-term management. A standard discounting rate of $3 \%$ per year was used. All costs were standardized to 2018 estimates as a common timeframe.

The total costs including upfront RT and the cost of normal tissue complication management were calculated

Table 1 The management and patient procedures related to the different normal tissue complications, as well as the estimated total cost of the different parts of managing the complications or the cost of the related patient procedure

\begin{tabular}{|c|c|c|c|}
\hline Normal tissue complication & Management/patient procedure & Estimated cost & Reference \\
\hline \multirow[t]{8}{*}{$\begin{array}{l}\text { Oral mucositis (grade } \geq 3 \text { ) or } \\
\text { Esophagitis (grade } \geq 3 \text { ) }\end{array}$} & $\begin{array}{l}\text { Fentanyl } 25 \mu \mathrm{g} / \mathrm{h} \text { patch (for } \\
6 \text { weeks) }\end{array}$ & $\$ 168.8$ & NADAC database \\
\hline & $\begin{array}{l}\text { Percocet } 325 \text { mg tablet (for } \\
6 \text { weeks) }\end{array}$ & $\$ 1514.1$ & NADAC database \\
\hline & Mucositis cocktail (for 6 weeks) & $\$ 37.0$ & NADAC database \\
\hline & Weekly IV hydration (for 4 weeks) & $\$ 154.2$ & $\begin{array}{l}2019 \text { Medicare Coding and Payment } \\
\text { Report }\end{array}$ \\
\hline & $\begin{array}{l}\text { PEG tube placement in 30\% of } \\
\text { cases }\end{array}$ & $\$ 5686^{*}$ & Callahan et al. [18] \\
\hline & $\begin{array}{l}\text { Emergency room visit in 15\% of } \\
\text { cases for oral mucositis and 10\% } \\
\text { of cases for esophagitis }\end{array}$ & $\$ 2096$ & $\begin{array}{l}2018 \text { Health Care Cost and Utilization }^{\text {Report }^{\dagger}}\end{array}$ \\
\hline & $\begin{array}{l}\text { In patient hospitalization in 10\% } \\
\text { of cases }\end{array}$ & $\$ 19,672$ & $\begin{array}{l}2018 \text { Health Care Cost and Utilization } \\
\text { Report }\end{array}$ \\
\hline & Loss of 1 month of work & $\$ 2718$ & $\begin{array}{l}\text { US Census Bureau Median per Capita } \\
\text { Income 2014-2018 }\end{array}$ \\
\hline \multirow[t]{2}{*}{ Dysphagia (grade $\geq 2$ ) } & Chronic PEG tube in $10 \%$ of cases & $\$ 18,836 /$ year* $^{*}$ & Callahan et al. [18] \\
\hline & $\begin{array}{l}\text { Stricture dilation in } 16 \% \text { of patients } \\
{[14,15]}\end{array}$ & $\begin{array}{l}\$ 1700 \text { (based on average Medicare } \\
\text { charges ranging from } \$ 1200 \text { to } \\
\$ 2200 \text { ) }\end{array}$ & $\begin{array}{l}\text { www.howmuchisit.org/esophageal } \\
\text {-dilation-cost/ (updated Aug 2018) }\end{array}$ \\
\hline $\begin{array}{l}\text { Hypothyroidism (elevated TSH with } \\
\text { or without T4/T3 changes requir- } \\
\text { ing hormone replacement) }\end{array}$ & $\begin{array}{l}\text { Levothyroxine hormone replace- } \\
\text { ment }\end{array}$ & $\begin{array}{l}\text { \$174.2/year (assuming } 70 \text { kg body } \\
\text { weight) }\end{array}$ & NADAC database \\
\hline \multirow[t]{2}{*}{ Xerostomia (grade $\geq 2$ ) } & Pilocarpine 5 mg (for 10 weeks) & $\$ 231$ & NADAC database \\
\hline & Cevimeline 30-45 mg (for 6 weeks) & $\$ 251.4$ & \\
\hline
\end{tabular}

\footnotetext{
* Adjusted for inflation from 1998 costs presented in the referenced paper to 2018 dollars

† https://healthcostinstitute.org/images/pdfs/HCCI_2018_Health_Care_Cost_and_Utilization_Report.pdf

‡ https://www.census.gov/quickfacts/fact/table/bronxcountybronxboroughnewyork,US/INC110218
} 
for 10,000 Monte Carlo samples for each of the 33 patient Results cases according to:

$$
\begin{aligned}
& \text { Cost }_{\text {Total }, \text { Photon }}=\operatorname{Cost}_{R T, \text { Photon }}+\sum_{i} \text { Cost }_{\text {Management }, i} \text { Event }_{i, \text { Photon }} P_{\text {Management }, i} \\
& \text { Cost }_{\text {Total,Proton }}=\text { Cost }_{R T, \text { Proton }}+\sum_{i} \text { Cost }_{\text {Management }, i} \text { Event }_{i, \text { Proton }} P_{\text {Management }, i}
\end{aligned}
$$

where $i$ represents each of the modeled normal tissue complications, Event represents whether or not that complication occurred in each Monte Carlo sample and $P_{\text {Management }}$ represents the proportion a certain management would be employed for complication $i$. For example, Levothyroxine would be employed in $100 \%$ of hypothyroidism cases whereas PEG tube placement is modeled to be needed in $30 \%$ of grade $\geq 3$ oral mucositis or esophagitis cases. For hypothyroidism and chronic PEG tube dependency following dysphagia, the cost of management is integrated over the patient's remaining life expectancy. Once the total costs and estimated QALYs for proton and photon therapy were calculated, incremental cost-effectiveness ratios (ICERs) for each sampled case were generated as:

$$
I C E R=\frac{\text { Cost }_{\text {Total,Proton }}-\text { Cost }_{\text {Total,Photon }}}{\text { QALY }}
$$

As such, ICERs represent the cost of sparing one full QALY with proton therapy. For cases with a zero or negative QALY difference photon therapy "dominates", and conversely, if the QALY difference is positive and the total cost of proton therapy is less than that of photon therapy, proton therapy dominates.

\section{Sensitivity analyses}

A one-way sensitivity analysis was performed to assess the impact of the assumed proportion of patients for whom dysphagia was chronic, or cleared up within 5 years in the QALY calculation. In the base case dysphagia was assumed chronic in $50 \%$ of cases and resolved within 5 years in $50 \%$. This was varied between respectively $0 \%$ and $100 \%$ in the sensitivity analysis.

We also performed a one-way sensitivity analysis of the cost of proton therapy to determine the impact on the cost-effectiveness results. In addition, a full sensitivity analysis was performed by varying the critical assumptions of the different components throughout the QALY and cost-effectiveness calculation steps as detailed in Table 2.
Incremental cost-effectiveness ratios (ICERs) were calculated for each of the 10,000 Monte Carlo samples for 33 individual patients, resulting in 330,000 total samples. In the base case, there is considerable variation in the calculated ICERs between patients, with median \$361,405/ QALY (IQR: \$45,453/QALY-\$1,556,948/QALY) for the entire cohort. The median ICER for patients $<65$ years old is $\$ 341,081 /$ QALY, compared to $\$ 399,533 /$ QALY for those who were $\geq 65$. There is a considerable difference in ICER based on p16-status with median \$516,297/QALY for patients with p16-negative tumors and \$234,201/ QALY for those with p16-positive tumors.

The variation in cost-effectiveness of proton therapy between patients is shown in Fig. 1, where the proportion of ICERs falling below certain cost-effectiveness thresholds are displayed, along with given patient characteristics. A larger proportion of sampled cases are considered cost-effective for patients receiving comprehensive nodal irradiation, compared to unilateral neck irradiation. This is related to $41.7 \%$ of sampled cases showing no difference in the estimated QALYs between photon and proton therapy for patients receiving unilateral nodal irradiation, as the risk of normal tissue complications is lower in this group. This is attributed to the lower risk of experiencing any of the modeled treatment complications (because of less normal tissue irradiated during unilateral treatment), leading to a lower likelihood that proton therapy would be cost-effective for these patients.

Our results show that proton therapy is more likely to be considered cost-effective for patients with p16-positive tumors treated with comprehensive nodal irradiation, with $42.4 \%$ of sampled cases considered costeffective at a threshold of $\$ 250,000 / \mathrm{QALY}$, compared to $26.6 \%$ of cases for those with p16-negative tumors treated with unilateral nodal irradiation. The variation between patients is further highlighted as the median ICER for the patient where proton therapy is most likely to be cost-effective is $\$ 54,477 / \mathrm{QALY}$, compared to $\$ 1,508,845$ / QALY for the patient where proton therapy is least likely to be cost-effective. 
Table 2 One-way and full sensitivity analyses performed as part of the cost-effectiveness evaluation

\begin{tabular}{|c|c|c|}
\hline Component & Base case & Variation in sensitivity analysis \\
\hline \multicolumn{3}{|l|}{ One-way sensitivity analysis } \\
\hline Proportion of chronic dysphagia & $\begin{array}{l}50 \% \text { chronic, } 50 \% \\
\text { resolved within } \\
5 \text { years }\end{array}$ & $\begin{array}{l}\text { Scenario 1: } 0 \% \text { chronic, } 100 \% \text { resolved within } 5 y \\
\text { Scenario 2: } 100 \% \text { chronic, } 0 \% \text { resolved within } 5 y\end{array}$ \\
\hline Cost of proton therapy (33 fx) & $\$ 36,659$ & $\begin{array}{l}\text { Scenario 1: } \$ 31,659 \\
\text { Scenario 2: } \$ 26,659 \\
\text { Scenario 3: } \$ 21,659\end{array}$ \\
\hline \multicolumn{3}{|l|}{ Full sensitivity analysis } \\
\hline \multicolumn{3}{|l|}{ QALY calculation } \\
\hline Hazard ratio for patients with > 10 pack-year smoking history & 1.73 & Normal distribution matching 95\% Cl 1.17-2.57 \\
\hline Quality-adjustment factors & & Beta distributions matching 95\% Cls: \\
\hline Dysphagia (grade $\geq 2$ ) & 0.83 & $0.70-0.93$ \\
\hline Esophagitis (grade $\geq 3$ ) & 0.66 & $0.35-0.90$ \\
\hline Xerostomia (grade $\geq 2$ ) & 0.82 & $0.72-0.90$ \\
\hline $\begin{array}{l}\text { Hypothyroidism (elevated TSH with or without T4/T3 changes requir- } \\
\text { ing hormone replacement) }\end{array}$ & 0.97 & $0.94-0.98$ \\
\hline Oral mucositis (grade $\geq 3$ ) & 0.06 & $0.01-0.15$ \\
\hline \multicolumn{3}{|l|}{ Cost-effectiveness calculation } \\
\hline $\begin{array}{l}\text { Proportion of oral mucositis or esophagitis cases requiring inpatient } \\
\text { hospital admission }\end{array}$ & $10 \%$ & Normal distribution matching 95\% Cl 0-20\% \\
\hline Proportion of oral mucositis cases resulting in emergency room visit & $15 \%$ & Normal distribution matching 95\% Cl 5-25\% \\
\hline Proportion of esophagitis cases resulting in emergency room visit & $10 \%$ & Normal distribution matching 95\% Cl 0-20\% \\
\hline Proportion of dysphagia cases requiring chronic PEG tube & $10 \%$ & Normal distribution matching 95\% Cl 0-20\% \\
\hline Proportion of patients receiving stricture dilation for dysphagia & $16 \%$ & Normal distribution matching 95\% Cl 11-21\% \\
\hline Cost of stricture dilation & $\$ 1700$ & Normal distribution matching 95\% Cl \$1200-\$2200 \\
\hline
\end{tabular}

More information about the components used in the quality-adjusted life years calculation can be found in ref [12]

\section{Sensitivity analyses}

Varying the assumption of dysphagia being chronic or cleared within 5 years did not substantially impact the results, with $35.2 \%$ of sampled cases considered costeffective at $\$ 250,000 /$ QALY in the base case, compared to $34.8 \%$ if resolved within 5 years and $35.6 \%$ if chronic.

Conversely, varying the cost of proton therapy had a significant impact on the results with a median ICER of $\$ 361,405 /$ QALY in the base case, compared to $\$ 244,351$ / QALY, \$133,234/QALY and \$26,394/QALY for an upfront proton therapy cost of $\$ 31,659, \$ 26,659$ and $\$ 21,659$, respectively. Similarly, the variation in proportion of sampled cases that would be cost-effective at different thresholds varies with proton therapy cost, as shown in Table 3.

In the base case, for patients with p16-positive tumors treated with comprehensive nodal irradiation proton therapy is estimated to be cost-effective in $\geq 50 \%$ of sampled cases for $8 / 9$ patients at $\$ 500,000 /$ QALY, compared to only $6 / 24$ patients who either have p16-negative tumors or receive unilateral irradiation. If the upfront cost of proton therapy was $\$ 31,659$ then this would instead be $8 / 9$ compared to $11 / 24$, respectively.
The results of the full sensitivity analysis are shown in Fig. 2 as the variation in total cost difference and QALY difference between proton and photon therapy for each of the Monte Carlo sampled cases. This shows a large variation in the sampled cases with a considerable proportion of data points to the right of the line that indicates an ICER equal to $\$ 100,000 / Q A L Y$, indicating proton therapy as cost-effective. The majority of data points are bunched together at a level closer to the $\$ 100,000 / Q A L Y$ line, where the estimated benefit from proton therapy is between 0 to 1.0 QALYs. In $22.9 \%$ of cases photon therapy dominates, i.e. there is no estimated benefit from proton therapy, mainly derived from sampled cases with no expected normal tissue complications. On the other hand, proton therapy dominates in $2.7 \%$ of sampled cases, where proton therapy has both a QALY benefit and are estimated to have a lower total cost compared to photon therapy. The two example patient cases in Fig. 2 where proton therapy is most likely or least likely to be cost-effective illustrate that for certain oropharyngeal cancer patients there is a considerable benefit from proton therapy with a majority of sampled cases to the right of the $\$ 100,000 / \mathrm{QALY}$ line, and conversely, little expected benefit for other patients. 


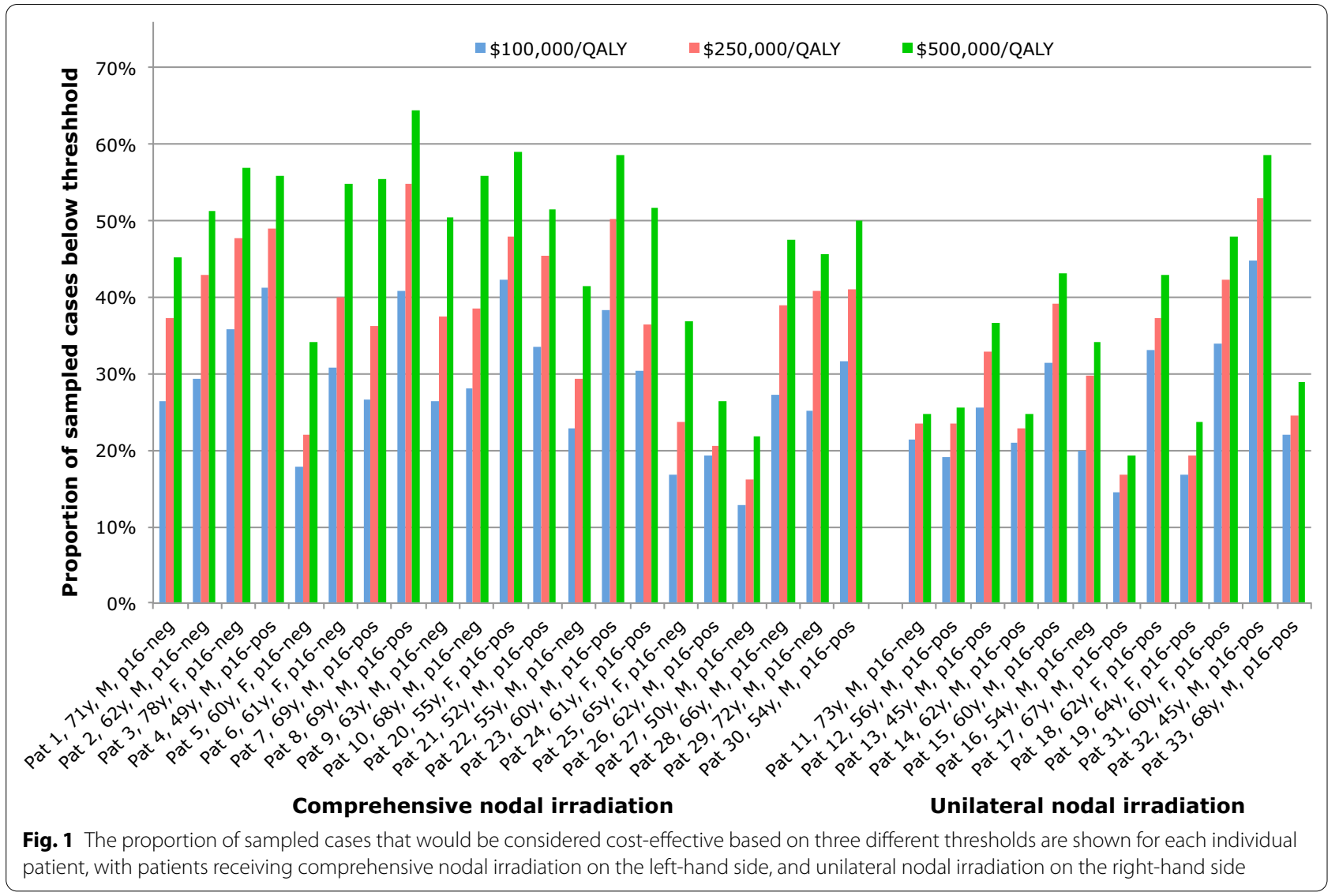

Table 3 The proportion of sampled cases that are considered cost-effective at different thresholds and varying cost of proton therapy for the whole patient cohort (for the patient where proton therapy is least likely to be cost-effective on the left-hand side of the parentheses, and most likely to be cost-effective on the right-hand side)

\begin{tabular}{|c|c|c|c|c|}
\hline \multirow[t]{2}{*}{ Cost-effectiveness threshold } & \multicolumn{4}{|l|}{ Proton therapy cost } \\
\hline & Base case: $\$ 36,659$ & $\$ 31,659$ & $\$ 26,659$ & $\$ 21,659$ \\
\hline$\$ 100,000 / Q A L Y$ & $\begin{array}{l}27.5 \% \\
(21.3,44.9 \%)\end{array}$ & $\begin{array}{l}32.6 \% \\
(22.7,51.3 \%)\end{array}$ & $\begin{array}{l}36.4 \% \\
(24.0,54.7 \%)\end{array}$ & $\begin{array}{l}56.0 \% \\
(34.6,66.8 \%)\end{array}$ \\
\hline$\$ 250,000 / \mathrm{QALY}$ & $\begin{array}{l}35.2 \% \\
(23.4,53.0 \%)\end{array}$ & $\begin{array}{l}39.4 \% \\
(24.2,56.2 \%)\end{array}$ & $\begin{array}{l}47.0 \% \\
(26.3,61.4 \%)\end{array}$ & $\begin{array}{l}65.7 \% \\
(51.8,70.8 \%)\end{array}$ \\
\hline$\$ 500,000 / Q A L Y$ & $\begin{array}{l}43.2 \% \\
(24.7,58.6 \%)\end{array}$ & $\begin{array}{l}48.4 \% \\
(27.2,62.3 \%)\end{array}$ & $\begin{array}{l}56.5 \% \\
(34.7,67.1 \%)\end{array}$ & $\begin{array}{l}70.5 \% \\
(61.8,72.5 \%)\end{array}$ \\
\hline
\end{tabular}

\section{Discussion}

Similar to the expected quality of life benefit, here we have shown that the cost-effectiveness of proton therapy varies strongly between individual patients. Proton therapy is estimated to be more cost-effective for patients with p16-positive tumors, and those treated with comprehensive nodal irradiation. This suggests that proton therapy would be most beneficial for oropharyngeal cancer patients who are expected to be at high risk of experiencing treatment-related normal tissue complications, and those who have a more promising cancer prognosis and therefore longer life expectancy. This type of modelbased approach provides an avenue for patient selection based on estimated clinical benefit, which can be weighted against the increased cost of treatment. This approach could also allow for testing the estimated benefits as patients are selected for one treatment vs. another, and could be re-calibrated as more clinical data becomes available. 

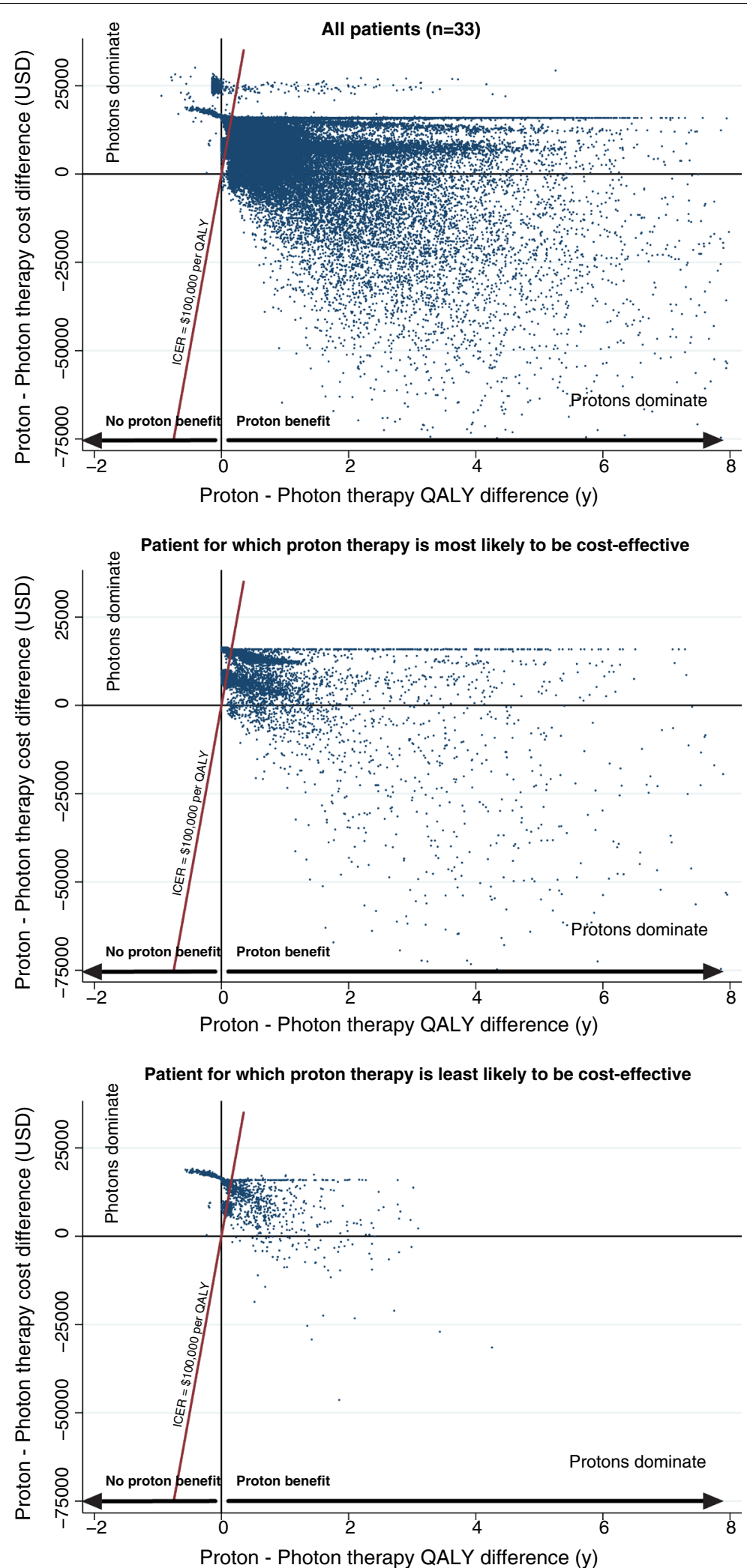

Fig. 2 The full sensitivity analysis showing cost and QALY differences between proton and photon therapy for all patients as well as for the patients for which proton therapy is most and lest likely to be cost-effective, respectively. Simulated cases to the right of the red line would be considered cost-effective at \$100,000/QALY whereas cases to the left would not 
The applied methodology estimates the cost-effectiveness of proton therapy based on dosimetric differences and corresponding NTCP estimates which then inform calculations on QALYs. Even when including the uncertainty in NTCP model parameters through all parts of the QALY calculation chain, the main factor driving the cost-effectiveness is the upfront cost of proton therapy. Clearly, lowering the cost to a level closer to that of current photon IMRT would generate more scenarios in which proton therapy is cost-effective at lower thresholds. Importantly though, for sub-groups of patients such as those with p16-negative tumors treated with unilateral neck irradiation, proton therapy may not be cost-effective even at a lower cost, as the estimated quality of life benefit is minimal for some of these patients.

In comparison to our results, Cheng et al. found that proton therapy delivered with IMPT would be cost-effective at $€ 80,000$ per spared QALY for 8 of $23 \mathrm{HNC}$ patients [7]. The reduction in the estimated risk of xerostomia and dysphagia they found with proton therapy is similar to our study, but the difference in upfront cost of the two treatments was somewhat smaller in their analysis. Conversely, a study by Ramaekers et al. [10] found that proton therapy was not cost-effective for HNC patients compared to photon IMRT. In their analysis they found an estimated average 0.10 QALYs spared with proton therapy, which is less than the average 0.37 QALY difference previously reported for our patient cohort [12]. This may be related to differences in the study populations or the fact that they only estimated xerostomia and dysphagia at 12 months as their endpoints, whereas we considered the risk of xerostomia, dysphagia, esophagitis, hypothyroidism and oral mucositis in this analysis.

One should also consider that both the upfront treatment cost and the costs related to managing complications may vary considerably between different countries and institutions. Thus the focus of this study was not to determine whether or not proton therapy is costeffective in general but to highlight the variability in estimated benefit and cost-effectiveness between individual patients, warranting individualized assessments when deciding whether to offer proton therapy. Another important limitation of this study is that not all possible treatment complications can be quantitatively modeled. Therefore, the estimates provided here may somewhat underestimate the cost-effectiveness of proton therapy, if the risks of non-modeled side effects are also reduced due to the lower integral dose from proton therapy. Based on the high costs of unplanned emergency room visits and hospitalizations, reducing these may be one of the key components of proton therapy cost-effectiveness. This is supported by a recent propensity-matched analysis showing reduced rates of severe toxicity and lower rates of unplanned hospitalizations in patients treated with proton therapy, compared to contemporary photon therapy [19].

Common to our study as well as those by others is the assumption that proton and photon therapy has the same treatment efficacy and probability of disease control. This is a critical assumption, as a reduction in loco-regional control would outweigh any benefit the patient receives from an improvement in OAR sparing. Encouragingly, results from preliminary clinical studies support this assumption of equal treatment efficacy between photon IMRT and proton IMPT [20, 21]. Key to this assumption is timely delivery of proton therapy, as delays in the time from diagnosis to treatment would negatively impact treatment outcome [22]. For the estimated benefit of proton therapy to withstand, efforts should be made to overcome existing logistical challenges related to proton therapy, in order to avoid delays in treatment delivery as compared to standard photon IMRT. Another recent study compared NTCP estimates between IMRT and pencil beam scanning proton therapy for a cohort of 30 oropharyngeal cancer patients [23]. Similar to our results they found that certain patients are expected to benefit greatly from proton therapy, with $\sim 15 \%$ reduction of xerostomia or dysphagia, whereas for others there was close to no difference in NTCP estimates between photons and protons.

\section{Conclusions}

Our results suggest that individualized decision-making is key when deciding whether to offer proton therapy for oropharyngeal cancer, which is supported by results from other studies as well. This strengthens the conclusion that proton therapy is unlikely to be cost-effective for all oropharyngeal cancer patients receiving definitive RT, and highlights the need to identify the patients with the most to gain from this treatment option. Furthermore, there is a difference in costs, societal willingness to pay and health system administration among different countries and this also needs to be considered when making decisions about which patients should receive proton therapy. Model-based decision-support systems and cost-effectiveness analyses such as the one presented here can help identify the patients for whom proton therapy is most likely to be beneficial, and serve as a decision-making aid for clinicians and health systems.

\section{Supplementary Information}

The online version contains supplementary material available at https://doi. org/10.1186/s13014-021-01745-1.

Additional file 1. Supplementary tables and figures. 


\begin{abstract}
Abbreviations
HNC: Head and neck cancer; RT: Radiation therapy; OARs: Organs at risk; IMRT: Intensity-modulated radiation therapy; QALYs: Quality-adjusted life years; IMPT: Intensity-modulated proton therapy; NTCP: Normal tissue complication probability; PEG: Percutaneous endoscopic gastrostomy; TSH: Thyroid stimulating hormone; NADAC: National Average Drug Acquisition Cost; ICERs: Incremental cost-effectiveness ratios.
\end{abstract}

\section{Acknowledgements}

This work was presented at the PTCOG 58th annual meeting in June of 2019 and was awarded the Michael Goitein award for best clinical work.

\section{Authors' contributions}

NPB, RK, WAT, CBS, MKG and SK were responsible for conceptualizing, planning and executing the study. MP and VG provided direct input in regards to proton therapy treatment planning for the study. NPB, RK and WAT generated and analyzed all data and results. All authors read and approved the final manuscript.

\section{Funding}

None.

\section{Availability of data and materials}

The datasets used and/or analyzed during the current study are available from the corresponding author on reasonable request.

\section{Ethics approval and consent to participate}

The study was approved by the institutional review board at the Albert Einstein College of Medicine with informed consent waived.

\section{Consent for publication}

Not applicable.

\section{Competing interests}

The authors declare that they have no competing interests.

\section{Author details}

${ }^{1}$ Institute for Onco-Physics, Albert Einstein College of Medicine, Bronx, NY 10461, USA. ${ }^{2}$ Department of Radiation Oncology, Montefiore Medical Center, Bronx, NY 10461, USA. ${ }^{3}$ Northwestern Medicine Chicago Proton Center, Warrenville, IL 60555, USA. ${ }^{4}$ Department of Family and Social Medicine, Albert Einstein College of Medicine, Bronx, NY 10461, USA. ${ }^{5}$ Department of Otorhinolaryngology - Head and Neck Surgery, Montefiore Medical Center, Bronx, NY 10461, USA. ${ }^{6}$ Department of Urology, Montefiore Medical Center, Bronx, NY 10461, USA. ${ }^{7}$ Department of Neurology, Albert Einstein College of Medicine, Bronx, NY 10461, USA

Received: 17 September 2020 Accepted: 2 January 2021

Published online: 21 January 2021

\section{References}

1. Ang KK, Harris J, Wheeler R, Weber R, Rosenthal DI, Nguyen-Tan PF, et al. Human papillomavirus and survival of patients with oropharyngeal cancer. N Engl J Med . 2010;363:24-35.

2. Ang KK, Zhang Q, Rosenthal DI, Nguyen-Tan PF, Sherman EJ, Weber RS, et al. Randomized phase III trial of concurrent accelerated radiation plus cisplatin with or without cetuximab for stage III to IV head and neck carcinoma: RTOG 0522. J Clin Oncol. 2014;32:2940-50.

3. Gregoire V, Langendijk JA, Nuyts S. Advances in radiotherapy for head and neck cancer. J Clin Oncol. 2015;33:3277-84.

4. Lim YJ, Wu HG, Kwon TK, Hah JH, Sung MW, Kim KH, et al. Long-term outcome of definitive radiotherapy for early glottic cancer: prognostic factors and patterns of local failure. Cancer Res Treat. 2015;47:862-70.

5. Nguyen-Tan PF, Zhang Q, Ang KK, Weber RS, Rosenthal DI, Soulieres D, et al. Randomized phase III trial to test accelerated versus standard fractionation in combination with concurrent cisplatin for head and neck carcinomas in the Radiation Therapy Oncology Group 0129 trial: longterm report of efficacy and toxicity. J Clin Oncol. 2014;32:3858-66.

6. Rosenthal DI, Harari PM, Giralt J, Bell D, Raben D, Liu J, et al. Association of human papillomavirus and p16 status with outcomes in the IMCL-9815 phase III registration trial for patients with locoregionally advanced oropharyngeal squamous cell carcinoma of the head and neck treated with radiotherapy with or without cetuximab. J Clin Oncol. 2016;34:1300-8.

7. Cheng Q, Roelofs E, Ramaekers BL, Eekers D, van Soest J, Lustberg T, et al. Development and evaluation of an online three-level proton vs photon decision support prototype for head and neck cancer-Comparison of dose, toxicity and cost-effectiveness. Radiother Oncol. 2016;118:281-5.

8. Jakobi A, Stutzer K, Bandurska-Luque A, Lock S, Haase R, Wack LJ, et al. NTCP reduction for advanced head and neck cancer patients using proton therapy for complete or sequential boost treatment versus photon therapy. Acta Oncol. 2015;54:1658-64.

9. Langendijk JA, Lambin P, De Ruysscher D, Widder J, Bos M, Verheij M. Selection of patients for radiotherapy with protons aiming at reduction of side effects: the model-based approach. Radiother Oncol. 2013;107:267-73.

10. Ramaekers BL, Grutters JP, Pijls-Johannesma M, Lambin P, Joore MA, Langendijk JA. Protons in head-and-neck cancer: bridging the gap of evidence. Int J Radiat Oncol Biol Phys. 2013;85:1282-8.

11. Sher DJ, Ringash J. Quality of life and value considerations in head and neck proton beam therapy: the holy grail at last, or the quest continues? Int J Radiat Oncol Biol Phys. 2016;95:40-2.

12. Brodin NP, Kabarriti R, Pankuch M, Schechter CB, Gondi V, Kalnicki $S$, et al. A quantitative clinical decision-support strategy identifying which patients with oropharyngeal head and neck cancer may benefit the most from proton radiation therapy. Int J Radiat Oncol Biol Phys. 2019;104:540-52.

13. Brodin NP, Kabarriti R, Garg MK, Guha C, Tome WA. Systematic review of normal tissue complication models relevant to standard fractionation radiation therapy of the head and neck region published after the QUANTEC reports. Int J Radiat Oncol Biol Phys. 2018;100:391-407.

14. Erkal EY, Canoglu D, Kaya A, Aksu G, Sarper B, Akansel G, et al. Assessment of early and late dysphagia using videofluoroscopy and quality of life questionnaires in patients with head and neck cancer treated with radiation therapy. Radiat Oncol. 2014;9:137.

15. Wang JJ, Goldsmith TA, Holman AS, Cianchetti M, Chan AW. Pharyngoesophageal stricture after treatment for head and neck cancer. Head Neck. 2012;34:967-73.

16. Vaidya B, Pearce SH. Management of hypothyroidism in adults. BMJ. 2008;337:a801.

17. Siddiqui F, Movsas B. Management of radiation toxicity in head and neck cancers. Semin Radiat Oncol. 2017;27:340-9.

18. Callahan CM, Buchanan NN, Stump TE. Healthcare costs associated with percutaneous endoscopic gastrostomy among older adults in a defined community. J Am Geriatr Soc. 2001;49:1525-9.

19. Baumann BC, Mitra N, Harton JG, Xiao Y, Wojcieszynski AP, Gabriel PE, et al. Comparative effectiveness of proton vs photon therapy as part of concurrent chemoradiotherapy for locally advanced cancer. JAMA Oncol. 2019;6:237-46.

20. Blanchard P, Garden AS, Gunn GB, Rosenthal DI, Morrison WH, Hernandez M, et al. Intensity-modulated proton beam therapy (IMPT) versus intensity-modulated photon therapy (IMRT) for patients with oropharynx cancer-a case matched analysis. Radiother Oncol. 2016;120:48-55.

21. Gunn GB, Blanchard P, Garden AS, Zhu XR, Fuller CD, Mohamed AS, et al. Clinical outcomes and patterns of disease recurrence after intensity modulated proton therapy for oropharyngeal squamous carcinoma. Int $J$ Radiat Oncol Biol Phys. 2016;95:360-7.

22. Schutte HW, Heutink F, Wellenstein DJ, van den Broek GB, van den Hoogen FJA, Marres HAM, et al. Impact of time to diagnosis and treatment in head and neck cancer: a systematic review. Otolaryngol Head Neck Surg. 2020;162:446-57.

23. Rwigema JM, Langendijk JA, Paul van der Laan H, Lukens JN, SwisherMcClure SD, Lin A. A model-based approach to predict short-term toxicity benefits with proton therapy for oropharyngeal cancer. Int J Radiat Oncol Biol Phys. 2019;104:553-62.

\section{Publisher's Note}

Springer Nature remains neutral with regard to jurisdictional claims in published maps and institutional affiliations. 